\title{
Reviewer acknowledgement 2015
}

\author{
Fernando De Benedetto ${ }^{1}$, Claudio F. Donner ${ }^{2}$ and Claudio M. Sanguinetti $3^{3^{*}}$
}

\section{Contributing reviewers}

The editors of Multidisciplinary Respiratory Medicine would like to thank all of our reviewers who have contributed to the journal in Volume 10 (2015).

\author{
Mohamed Abdelrahim \\ Egypt \\ Hafiz Omer Ahmed \\ United Arab Emirates \\ Sara Albolino \\ Italy
}

Nicolino Ambrosino

Italy

Bruno Balbi

Italy

Salvatore Bellofiore

Italy

Giorgio Bertolotti
Italy

Gian Luca Biscione

Italy

Claudio Bruschi

Italy

Stefano Carlone

Italy

Piero Ceriana

Italy

Kusum S Chand

India

Lorenzo Corbetta

Italy

George Cremona

Italy

\author{
Roberto Dal Negro \\ Italy
}

Gennaro D'Amato

Italy

Silvestro Ennio D'Anna

Italy

Fernando De Benedictis

Italy

Aliye Esmaoglu

Turkey

Paola Faverio

Italy

Giovanni Ferrara
Italy

Giovanni Fontana

Italy

Silvia Forte

Italy

Giorgio Fumagalli

Italy

Gisela Soboll Hussey

USA

Cicchitto Gaetano

Italy

Junkal Garmendia

Spain

Stefano Gasparini

Italy
Peter Gay

USA

\section{Claudio Henriquez}

Chile

Giuseppe Insalaco

Italy

Alicja Iwaszko

Poland

Vangelis Karalis

Greece

Nora Kovats

Hungary

Philip Chi Lip Kwok

Hong Kong

Maria Laucho-Contreras

USA

Mirco Lusuardi

Italy

Mauro Maniscalco

Italy

Stefano Marinari

Italy

Christian Nagel

Germany

Margherita Neri

Italy

Gregorino Paone

Italy

* Correspondence: mrm@biomedcentral.com

${ }^{3}$ Quisisana Clinical Center, Rome, Italy

Full list of author information is available at the end of the article 
Girolamo Pelaia

Italy

Pietro Pirina

Italy

William A Prescott Jr

USA

Richard Riker

USA

Silvia Rossi Ferrario

Italy

Yogesh Saini

USA
Manuel Sanchez Solis

Spain

Antonio Sanna

Italy

Monika Szturmovwicz

Poland

Martin Tobin

USA

Roberto Torchio

Italy

Antoni Torres

Spain

\section{Giovanni Viegi}

Italy

Michele Vitacca

Italy

Alessandro Zanasi

Italy

Pietro Zanon

Italy

Author details

${ }^{1}$ Specialisation School, University G. D'Annunzio,

Chieti, Italy. ${ }^{2}$ Mondo Medico Clinic, Borgomanero,

Italy. ${ }^{3}$ Quisisana Clinical Center, Rome, Italy. 